\title{
Announcing Regenerative Engineering and Translational Medicine
}

BMES Fellow and Board Member Cato Laurencin (UConn) is pleased to announce the launch of Regenerative Engineering and Translational Medicine (RETM), published by Springer and the newly formed Regenerative Engineering Society. Dr. Laurencin is the journal's Editor-in-Chief, and the Executive Advisory Editors are Robert Langer (MIT), Robert M. Nerem (Georgia Tech), Nicholas Peppas (University of Texas at Austin), C.N.R. Rao (Jawaharlal Nehru Centre), and Xingdong Zhang (Sichuan University).

RETM is an international journal covering Convergence of the disciplines of advanced materials science, stem cell research, the physical sciences, and areas of developmental biology and clinical translation. This Convergence brings exciting opportunities to translate bench-top research into bedside methods, allowing the possibility of moving beyond maintaining or repairing tissues to regenerating them. All research articles in RETM feature a lay abstract highlighting the relevance and future impact for patients, government and other health officials, and members of the general public. Bridging the gap between the lab and the clinic, the journal also serves as a dedicated platform for showcasing translational research that brings basic scientific research and discoveries into clinical methods and therapies, contributing to the improvement of human health care.

The Journal is by subscription, but will be free to all for the first two years of publication.

"We are now just over a quarter of a century out from the beginning of the field of tissue engineering, and Regenerative Engineering and Translational Medicine is the first journal to challenge us to think beyond traditional comfort zones and Converge fields," says Dr. Laurencin.

The journal's first papers have been published and can be read online on SpringerLink. RETM welcomes submissions from BMES members, and more information can be found at www.springer.com/40883. Convergence papers on instructive biomaterials, stim-
Volume $1 \cdot$ Number 1

\section{Regenerative Engineering and}

\section{Translational Medicine}

An Official Journal of the

Regenerative Engineering Society

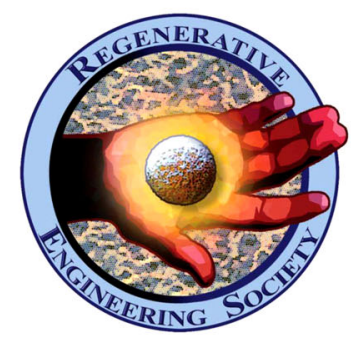

Springer

$40883 \cdot$ elSSN 2196-8837 1(1) $001-000$ (2015)

uli-responsive biomaterials, micro- and nano-patterning for regenerative engineering, novel approaches toward regeneration, bioreactor systems and rapid prototyping and bioprinting approaches are particularly welcome. 